\title{
Management of a kidney stone in ectopic pelvic kidney with Extracorporeal Shockwave Litothripsy: Description of a case and revision of literature
}

\author{
Carmelo Agostino Di Franco ${ }^{1}$, Maurizio Burrello ${ }^{1}$, Francesco Guzzardi ${ }^{1}$, Eva Intagliata ${ }^{1}$, \\ Irina Oxenius ${ }^{1}$, Lavinia Galvagno ${ }^{2}$, Calogero Cordaro ${ }^{1}$ \\ ${ }^{1}$ Department of Urology, S. Elia Emergency Hospital, Caltanissetta, Italy; \\ ${ }^{2}$ Translation and Language Revision Service, Enna, Italy.
}

\begin{abstract}
Summary Urolithiasis is one of the most frequent disease in the population and it represents one of the most frequent causes of access to emergency department. In addition. congenital anomalies occur more often in the kidney than in any other organ and the association of both renal abnormalities and stones is of clinical relevance. In this report, we discuss a case of a women with pelvic ectopic kidney affected by a large pyelic stone treated with ESWL (Extracorporeal Shockwave Lithotripsy).
\end{abstract}

KEY WORDS: Pelvic ectopic kidney; Urinary calculi; Extracorporeal Shockwave Lithotripsy.

Submitted 24 November 2019; Accepted 3 January 2020

\section{INTRODUCTION}

Urolithiasis is one of the most frequent diseases in the population and it represents one of the most frequent causes of access to the emergency department. For patients affected by kidney anomalies, stone treatment could be a strong challenge for the Urologist. In addition, Extracorporeal Shockwave Lithotripsy (ESWL), is considered one of the first-line option treatment for kidney stones. In particular, Retrograde Intrarenal Surgery (RIRS) is not always available in all departments and it is associated to a higher complication rate than ESWL. However, urinary stasis that usually is associated with kidney anomalies, could interfere with clearance of stone fragments after shockwave lithotripsy.

In this report, we discuss a case of a woman with pelvic ectopic kidney affected by a large pyelic stone treated with ESWL.
\end{abstract}

\section{Case report}

We describe the case of a 63-years woman who presented to our emergency department with fever $39^{\circ} \mathrm{C}$ and abdominal pain. Blood samples showed leukocytosis (WBC 21000) and increased C-reactive protein, normal kidney function. Computed tomography (CT)-scan showed a right pelvic ectopic kidney with hydronephrosis and a large pyelic stone around 19 millimeters (Figure 1). Radiologist described increased peripyelic fat density secondary to phlogistic process. The patient was subjected to ureteral double J stenting into the ectopic kidney and bladder catheter with emission of pyuria. We treated the septic status with antibiotic (piperacillin/ tazobactam) and parenteral hydration. The patient was monitored with blood samples that showed a rapid improvement. In the first post-operative day the patient was afebrile. The patient was affected by diabetes mellitus type II controlled with diet and she did not take any drug. After one month, the patient was subjected to first ESWL treatment on the ectopic kidney stone; ureteral double J stent was maintained inside.

In total, we performed two ESWL treatments. We monitored stone changes after the first ESWL with radiographic scans (Figure 2).

The position on the treatment-table was prone and we performed fluoroscopic control during the procedure. The shockwave lithotripsy was performed with a Sonolith ${ }^{\circledR}$ device using electro-conductive shock head module. In both the procedures, we administered 3000 shocks, middle energy was $657 \mathrm{~J}$, frequency $2 \mathrm{~Hz}$.

Fluoroscopy time was 02 minutes and 25 seconds with X-ray dose $3687 \mathrm{mGy} / \mathrm{cm}^{2}$ in first treatment and $02 \mathrm{~min}$ utes and 48 seconds with X-ray dose $3702 \mathrm{mGy} / \mathrm{cm}^{2}$ in second treatment. Procedures were well tolerated; the patient did not need any antalgic drug during or after the procedure. No immediate complications were reported. We reported macrohaematuria during the first operative day in both procedures with spontaneous resolution and occasional dysuria during the two months with ureteral stent. After the second treatment, we performed outpatient ultrasound check and abdominal CT-scan. CT-scan showed complete stone free ectopic kidney as shown in Figure 3.

We removed ureteral stent after CT-scan check, two weeks from latest ESWL treatment. The patient was enrolled to our outpatient service and metabolic study.

\section{Discussion}

The introduction of ESWL in 1980 revolutionized the treatment of kidney stones. It is a less invasive treatment for kidney stones, with low rate of complications and well accepted by patients. Therefore, it is now used for almost $80 \%$ of patients with urinary stones. However, 

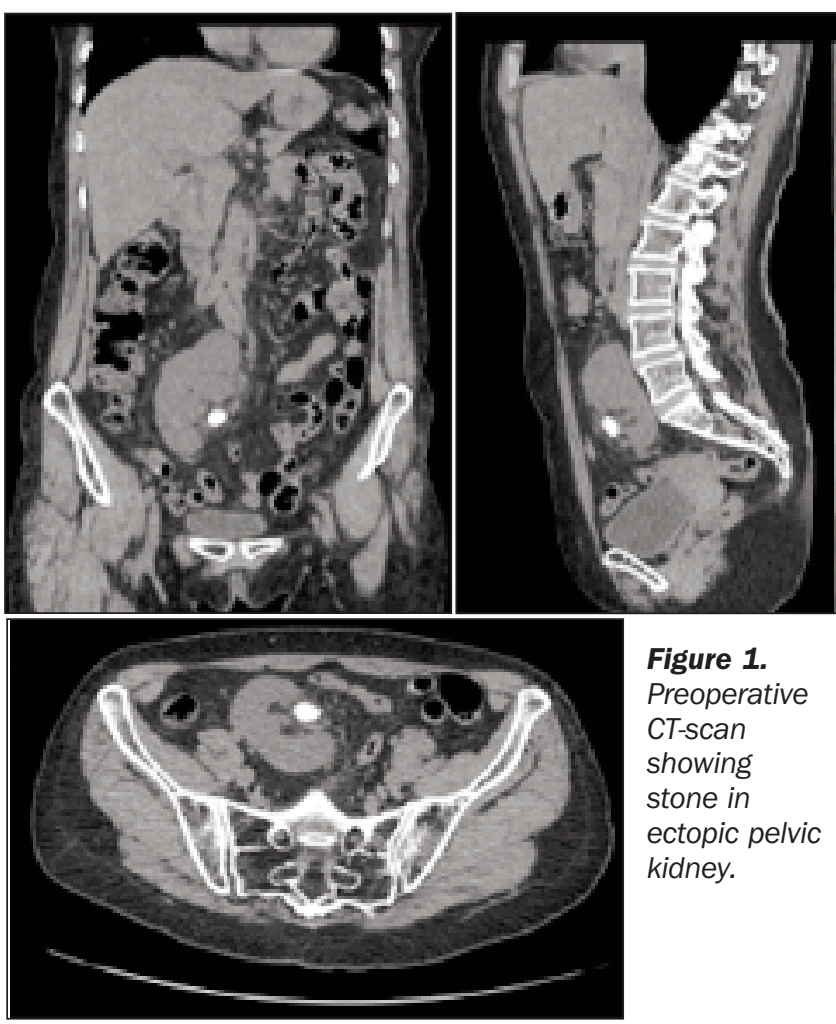

Figure 1. Preoperative CT-scan showing stone in ectopic pelvic kidney.

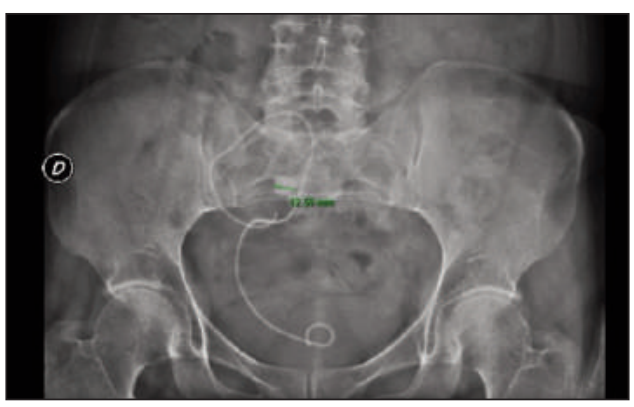

Figure 2.

$R x$ - check showing stone modification after the $1^{\text {st }}$

ESWL

treatment.

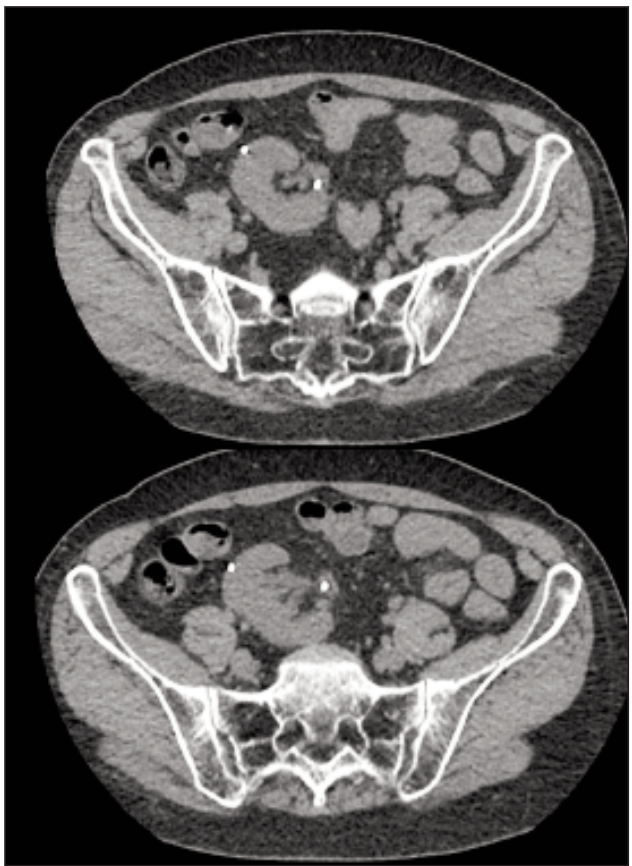

Figure 3.

CT-scan after the $2^{\text {nd }} E S W L$ treatment showing 100\% stonefree rate with ureteral $J$ stent inside. today other minimally invasive techniques such as RIRS or mini-PERC (Percutaneous Stone Surgery) represent good solutions for kidney stone treatment although they are not always available in all centers especially in secondary hospitals.

As reported in literature, ESWL can obtain good results in patients with malformed kidney, with an overall stonefree rate of $71.77 \%$ (1). For stones smaller than $1 \mathrm{~cm}$, the stone-free rate was higher (up to 96.1\%) demonstrating the importance of stone size for a successful treatment.

Brad et al. (2) reported retrospectively ESWL results in a population with renal malformations; in their study average stone size was $10.23 \mathrm{~mm}$ and stone free-rate was $71.7 \%$.

They reported two cases of subcapsular hematoma (1.7\%), two cases of acute pyelonephritis (1.69\%) and two cases of haematuria which needed bladder washout and catheterization. Overall complication rate in their report was $13.56 \%$. In addition, the most severe complication is renal haematoma: it seems to be favoured in patients suffering of hypertension, liver diseases or anticoagulation drug use (2)

In literature we found some studies related to the use of the ureteral stent in association with ESWL. Common idea is that ureteral stents compromise the results of ESWL with a difference of around 22\% in the stone-free rate in favour of non-stented patients as ureteral stents interfere with stone clearance because the presence of stent hinders the passage of fragments after ESWL.

In particular, a recent paper by Ahmad et al. (3) reports that stone-free rate within patient without double J stent was $81,8 \%$ and within patients with JJ stent was $16.7 \%$ $(\mathrm{p}<0.05)$.

Regarding stone-free rate in the management of stones in anomalous kidneys with ESWL, in literature some studies are reported, with a range from $31 \%$ to $100 \%$. Brad et al. (2) reported stone-free rate of $71.7 \%$ in 118 patients with kidney abnormalities and a medium stone size of $10.2 \mathrm{~mm}$.

The study of Al-Tawheed et al. (4) reported stone-free rate 83,9\% with 9 patients with ectopic kidney an average stone-size of $1.5 \mathrm{~cm}$. In Ahmad et al. study (3) 50 patients with kidney malformations were evaluated and 22\% of patients had ectopic kidney. In ectopic kidney group, stone-free rate was 100\% with ectopic abdominal kidney, $75 \%$ with ectopic lumbar kidney and 0\% with ectopic pelvic kidney.

Stone size was relevant, in particular stone-free rate was $74 \%$; in patients with stone size below $1 \mathrm{~cm}$, stone-free rate rose up to $91,6 \%$ demonstrating the importance of stone size for a successful ESWL treatment.

In our report, we describe a successful ESWL treatment in a large stone (19 $\mathrm{mm}$ ) in ectopic pelvic kidney. In our case patient had ureteral stent because she was first treated for septic status. However, in our routine we usually place the stent to patients with kidney calculi greater than $1.5 \mathrm{~cm}$.

\section{Conclusions}

ESWL is a good treatment for kidney stones also in malformed kidneys achieving a good stone-free rate. 
However, the success of ESWL depend on many factors, in particular stone size, stone density and kidney position. The stone-free rate is related to patient compliance: in our case the patient was very collaborative and she did not need any analgesic drug.

In addition, ESWL treatment in selected cases could be an alternative to other stone treatments such us RIRS or percutaneous nephrolithotomy (PCNL).

\section{REFERENCES}

1. Tunc L, Tokgoz H, Tan MO, et al. Stones in anomalous kidneys: results of treatment by shock wave lithotripsy in 150 patients. Int J Urol. 2004; 11:831-6.

2. Brad A, et al. The place of ESWL in the treatment of urinary stones in patients with renal malformations." Romanian Journal of Urology. 2016; 15:28-32

3. Ahmad MNA, Hussein Abdallah Galal HA, Ayman Kotb Koritinah AK. Outcome of extracorporeal shockwave lithotripsy in congenital malformed kidneys. The Egyptian Journal of Hospital Medicine 2019; 76:3963-3967.

4. Al-Tawheed, AR, Al-Awadi KA., Kehinde EO, et al Treatment of calculi in kidneys with congenital anomalies: an assessment of the efficacy of lithotripsy. Urol Res. 2006; 34:291-298.

\section{Correspondence}

Carmelo Agostino Di Franco, MD (Corresponding Author) carmelo_difranco@tiscali.it

Maurizio Burrello

Francesco Guzzardi

Eva Intagliata

Irina Oxenius

Calogero Cordaro

donc86@tiscali.it

Department of Urology, S.Elia Emergency Hospital, Caltanissetta (Italy)

Lavinia Galvagno

Translation and Language Revision Service, Enna (Italy) 